\title{
Experiencia mexicana de la medicina de urgencias en las misiones análogas a Marte
}

\author{
Nancy Labastida-Mercado* \\ Hospital General Dr. Gaudencio González Garza del Centro Médico Nacional La Raza, IMSS, Ciudad de México, México; Mars Academy, Los \\ Ángeles, California, EE.UU.
}

\begin{abstract}
Resumen
Las misiones análogas son simuladores en ambientes extremos para el cuerpo humano en misiones espaciales de larga duración, formadas por grupos de científicos. La Mars Academy USA creó el primer programa de Misiones de Médicos Astronautas Análogos, una serie de misiones de simulación que se centran en innovaciones en medicina espacial, biomédica y biotecnología, para capacitar científicos como astronautas análogos en un hábitat donde puedan desarrollar e innovar formas de apoyar futuras exploraciones y asentamientos humanos en Marte y la Luna, mediante tecnologías exponenciales, como realidad virtual, impresión $3 D$, herramientas genéticas, tecnologías de telemedicina, robótica y drones. El presente artículo describe la experiencia de un médico mexicano residente de la especialidad de Medicina de Urgencias en la primera tripulación de médicos astronautas análogos del proyecto Mars Medics.
\end{abstract}

Palabras clave: Medicina de emergencia. Medicina aeroespacial. Ambientes extremos. Evaluación de la tecnología biomédica.

\section{Mexican experience of Emergency Medicine in Mars analogous missions}

\section{Abstract}

The analogous missions are simulators in extreme environments for the human body in long-term space missions, made up of groups of scientists. Mars Academy USA created the Mars Medic Analog Astronaut Training Program, a series of simulation missions that focus on innovations in space medicine, biomedicine and biotechnology, to train scientists as analogous astronauts in an environment of developing and innovating ways, to support future explorations and human settlements on Mars and the Moon, through exponential technologies such as virtual reality, 3D printing, genetic tools, telemedicine technologies, robotics and drones. This article describes the experience of a Mexican resident in Emergency Medicine in the first crew of analogous astronaut doctors of the Mars Medics project.

Key words: Emergency medicine. Aerospace medicine. Extreme environment. Technology assessment biomedic.

\section{Correspondencia:}

*Nancy Labastida-Mercado

Jacarandas y Vallejo, $\mathrm{s} / \mathrm{n}$ 


\section{Introducción}

La medicina aeroespacial es una rama de la Medicina que se encarga de estudiar las enfermedades y trastornos del cuerpo humano asociados con la exposición a medios ambientes extremos como la aviación, el submarinismo y el espacio. La medicina aeroespacial nació en la década de 1950 y desde entonces hasta el día de hoy la duración de los periodos de tiempo en ambientes extremos ha aumentado; por ejemplo, la permanencia en la Estación Espacial Internacional, donde el astronauta Gennady Padalka pasó 878 días en cinco misiones diferentes (actualmente posee el récord del mayor tiempo de estancia en el espacio), lo que ha llevado a esta rama médica a estudiar los efectos a largo plazo debidos a la microgravedad, la radiación y el aislamiento'. La medicina basada en evidencia, la investigación y la experiencia clínica han sido los principales impulsores del esfuerzo interminable para asegurar la vida de los astronautas y comprender cómo el entorno espacial influye en la fisiología humana. Así es como se diseñaron los proyectos denominados misiones análogas.

\section{Desarrollo del tema}

Las misiones análogas son simuladores en ambientes extremos para el cuerpo humano, como desiertos y montañas, donde se realizan réplicas de actividades humanas para misiones de larga duración, por ejemplo, para probar nuevos equipos o alimentos, y están formadas por grupos de científicos que deben sobrevivir en condiciones extremas durante varios días ${ }^{2}$. Los hábitats donde realizan estas misiones análogas se eligen por su ubicación geográfica y por las características que poseen cada uno de ellos, por ejemplo, la isla Devon en Canadá, que está a una latitud de $75^{\circ}$ al Norte $^{3}$, lo que proporciona un resplandor solar similar al ecuador marciano, porque las altitudes son equivalentes a la baja presión de la atmósfera de Marte ${ }^{4}$; 0 Death Valley, al sureste de California (en EE.UU.), que cuenta con el punto de elevación más bajo (-86 m sobre el nivel del mar) en toda América del Norte, y donde existe el récord de la temperatura más alta registrada en el mundo $\left(56.7^{\circ} \mathrm{C}\right)$, lo cual favorece que sea un área geográfica para crear un hábitat similar a la superficie marciana ${ }^{4}$.

Mars Academy USA (MAU) fue fundada en el año 2016 por la Dra. Susan Ip-Jewell, actualmente su presidenta y directora, quien creó un nuevo paradigma en el aprendizaje mediante el uso de tecnologías exponenciales, aprendizaje basado en simulación y entretenimiento educativo. La Dra. Jewell es una comandante experimentada de muchas misiones análogas, que ha formado y entrenado a varios equipos para expediciones. Fue la creadora del primer programa de Misiones de Médicos Astronautas Análogos, una serie de misiones de simulación que se centran en innovaciones en medicina espacial, biomedicina y biotecnología, con la misión de capacitar científicos como astronautas análogos para trabajar juntos en un entorno transdisciplinario y multicultural (Fig. 1). Las misiones MAU Mars Medics (MMM) se centran específicamente en innovaciones para exploraciones en medicina espacial, astrobienestar, producción de alimentos espaciales y nutrición. Las tripulaciones de esta serie de misiones análogas tienen el objetivo de desarrollar e innovar formas de apoyar futuras exploraciones y asentamientos humanos en Marte y la Luna, por medio de tecnologías como realidad virtual, impresión 3D, herramientas genéticas, tecnologías de telemedicina, robótica y drones ${ }^{5,6}$.

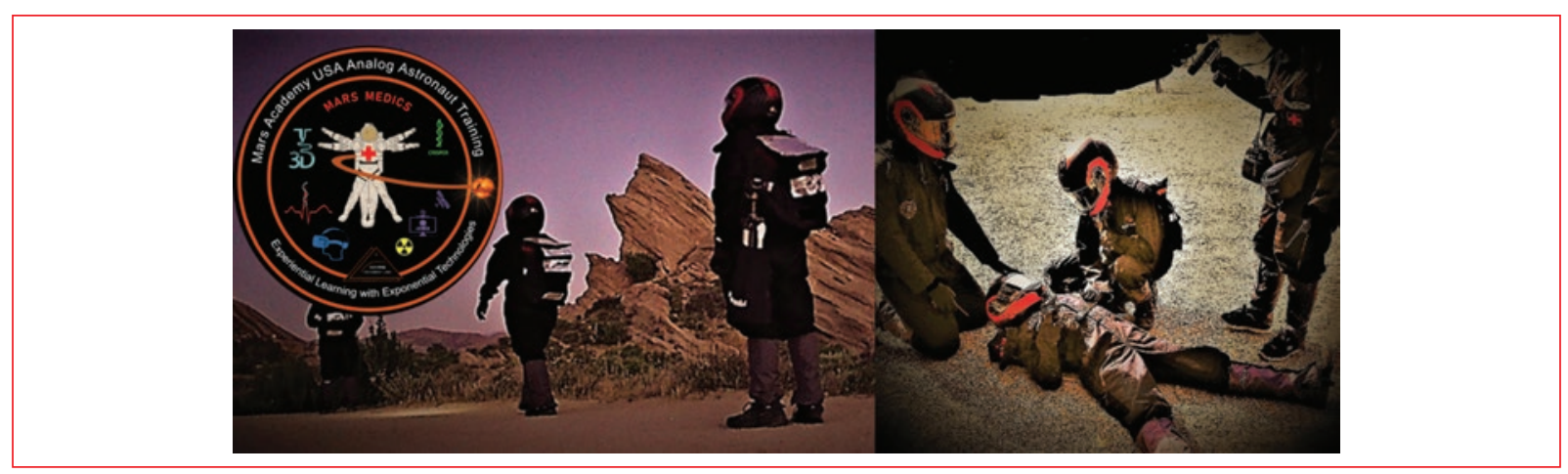

Figura 1. Imágenes de actividades médicas extravehiculares. Esquina superior izquierda: parche de la Mars Academy USA Analog Astronaut Training. 


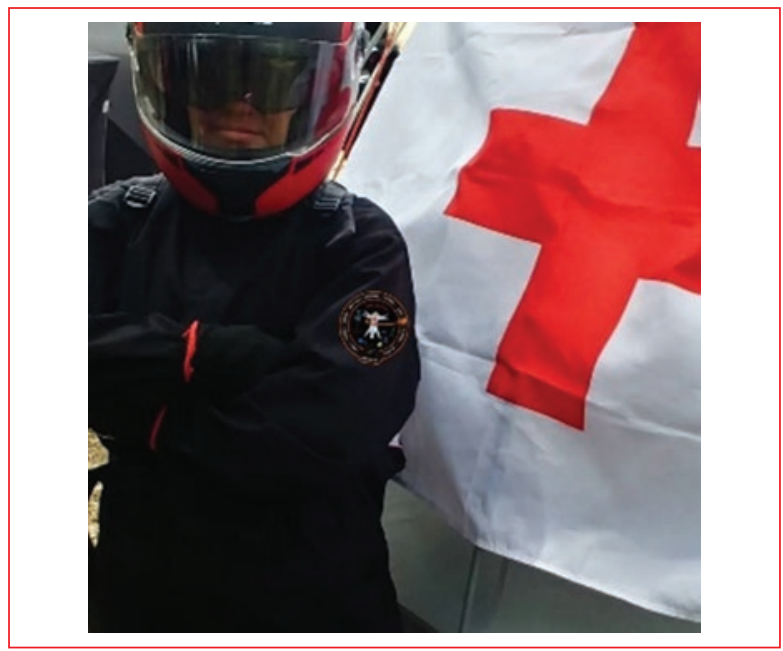

Figura 2. Dra. Nancy L. Mercado, Crew Surgeon of Mars Medics I, al fondo Space Clinic de la Mars Academy USA.

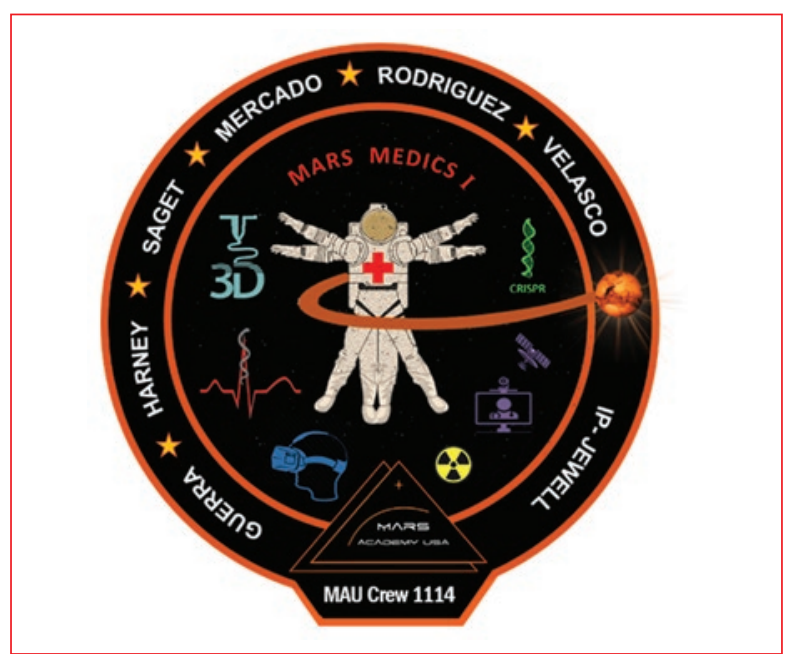

Figura 3. Parche de la Primera Misión de Médicos Astronautas Análogos Crew 1114-Mars Medics I de la Mars Academy USA (MAU). Miembros de la Tripulación: Dr. Jesús Guerra, Dra. Mariya Harney, Dra. Nancy L. Mercado, Dr. Eduardo Rodríguez, Jay Velasco, Dr. Jeremy Saget (Comandante de la tripulación), Dra. Susan Ip-Jewell (Presidente y Director de la MAU).

En mi último grado como residente de Medicina de Urgencias fui seleccionada por la MAU para formar parte de la primera tripulación de médicos astronautas análogos Mars Medics I - Crew 1114, cuya formación se llevó a cabo en el desierto de California (EE.UU.) durante la primavera del año 2018 (Fig. 2 y 3). La tripulación 1114 estuvo formada por: el Dr. Jesús Guerra-Rivera, último médico mexicano egresado del posgrado de Medicina Aeroespacial de la Wright State
University y coautor de diversas publicaciones en Medicina Aeroespacial; la Dra. Mariya Harney, especialista en Psiquiatría; el Dr. Eduardo Rodríguez, médico del Programa de Investigación Suborbital Polar Suborbital Science in the Upper Mesosphere; el Dr. Jeremy Saget, instructor de Zero-G, Parabolic Flights de la European Space Agency; Jay Valesco, astronauta análogo pionero de $M A U$, y la Dra. Ip-Jewell. A una servidora, como miembro en su momento del Colegio Mexicano de Medicina Aeroespacial y de la Asociación de Medicina de Aviación y del Espacio, se me asignó estar a cargo de las actividades médicas extravehiculares, en el estudio de cómo estas actividades se ven influenciadas en la naturaleza del trauma y el potencial de supervivencia de los astronautas análogos, así como los procedimientos y protocolos de búsqueda y rescate, la evaluación de las pruebas para la implementación de drones y robots para sistemas de rescate ante desastres sobre la superficie planetaria (para beneficiar y apoyar las emergencias médicas terrestres). También colaboré en los entrenamientos de simulación de teleanestesia-telecirugía y su implementación para equipos de respuesta inmediata ante una emergencia médica en las actividades extravehiculares para astronautas análogos no médicos.

Las tareas asignadas a cada uno de los miembros de la tripulación son encaminadas para que inspiren e interesen a futuras generaciones de astronautas análogos en la exploración espacial y en el futuro asentamiento de Marte, la Luna y otros planetas. Así es como MAU ha participado en la convención International Space Station Research \& Development, un evento celebrado en San Francisco y patrocinado por la National Aeronautics and Space Administration (NASA) y el Center for Advancement of Science in Space (CASIS). Otros reconocimientos importantes que ha recibido este proyecto han sido en el Next Generation Suborbital Researchers Conference (NSRC2018) en Broomsfield, EE.UU.; en la Aerospace Medical Association 2018 en Texas, EE.UU.; el International Astronautical Conference 2018 en Bremen, Alemania, y el NASA Space App Challenge en Pasadena, EE.UU. ${ }^{5,6}$.

Esta experiencia me permite dar a conocer por medio de esta breve reseña, que la Medicina de Urgencias no tiene límites. Los incidentes médicos más frecuentemente reportados en los programas espaciales han implicado lesiones traumáticas menores limitadas a la piel, y aunque se han producido numerosas muertes catastróficas, no se han realizado evacuaciones de emergencia por traumas graves, incluso hay un vehículo de emergencia para la Estación Espacial 
Internacional, la Soyuz, que es capaz de regresar a la Tierra si esta emergencia ocurriera con un tiempo estimado de media hora.

\section{Conclusión}

La Medicina de Urgencias tiene aún más problemas a los que no nos hemos enfrentado, como la alta probabilidad de presentar un trauma o una emergencia médica en una misión de larga duración, situación en la cual, a diferencia de la permanencia en la Estación Espacial Internacional, no existiría la posibilidad de facilitar una salida de emergencia con retorno al planeta Tierra en menos de una hora, y actualmente es una de las mayores preocupaciones de las agencias espaciales. Una lesión traumática grave en una misión de este tipo requeriría iniciar un tratamiento en el trayecto de vuelo, debido a que el regreso a la Tierra no será rápido, existen muchos riesgos y alteraciones fisiológicas sumadas al aislamiento de larga duración. Se requerirá un médico con el perfil de un urgenciólogo bien entrenado a bordo de las tripulaciones, que tenga capacidad quirúrgica y que pueda resolver problemas médicos e improvisar con el equipo médico disponible. Hasta el momento ninguna tecnología de telemedicina, por más avanzada que sea, es capaz de salvaguardar las vidas de astronautas en la proyección de estas misiones de larga duración, sin embargo, muchas de las implementaciones tecnológicas actualmente están ya disponibles en la Tierra, como lo han hecho hasta el momento la combinación de la cirugía entre realidad virtual y el equipo médico creado por medio de impresión en 3D, en el esfuerzo de satisfacer las necesidades de atención médica que permiten un mejor acceso a sistemas de salud en los lugares más remotos y de difícil acceso en el mundo.

La MAU ha permitido que una comunidad de científicos de todo el mundo trabajemos juntos para perfeccionar proyectos de misiones análogas y logremos el desarrollo de ideas que también contribuyan a mejorar la vida en la Tierra, con un futuro prometedor para las Ciencias de la Salud, especialmente la Medicina de Urgencias.

\section{Conflicto de intereses}

Declaro que no existe potencial conflicto de interés, incluyendo cualquier relación financiera, personal o de otra naturaleza con alguna persona u organización que pudiera influir, o que pudiera percibirse como influyente en las contribuciones científicas a este artículo.

\section{Bibliografía}

1. Landon LB, Slack KJ, Barret JD. Teamwork and collaboration in long duration space missions: Going to extremes. Am Pshychol. 2018; 73(4):563-75.

2. Bell ST, Brown SG, Abben DR, Outland NB. Teamwork composition issues for future space exploration: A review and directions for future research. Aerosp Med Hum Perform. 2015;86(6):548-56.

3. Koerner RM. Devon Island ice cap: core stratigraphy and paleoclimate. Science. 1977;196(4285):15-8.

4. Cabrol NA. The coevolution of life and environment on Mars: An ecosystem perspective on the robotic exploration of biosignatures. Astrobiology. 2018;188(1):1-27.

5. Ip-Jewell. Experiential learning in exponential technologies [Internet]. California, EE.UU.: Mars Academy USA. (fecha de acceso: 15 de diciembre de 2018). Disponible en: https://www.marsacademyusa.com/publications.

6. Ip-Jewell. Experiential Learning in Exponential Technologies. California, EE.UU.: Mars Academy USA. (fecha de acceso: 15 de diciembre de 2018). Disponible en: https://www.marsacademyusa.com/past-missions 